Imre Gábor Nagy

\title{
Die Vermögenswirtschaft der Stadt im Zeitalter des Dualismus
}

\begin{abstract}
In the annuel budgets of the city of Pécs between 1872 and 1914, revenues from city property were divided into five groups. The first group included revenues from the city's property - the hundreds of acres of Megyer-puszta, urban pastures, urban factories, and urban buildings. The second group included revenues from the city's 4,262 cadastral hungarian acres forests. The third group included interest on the city's cash and securities. The fourth group included excise, duties and fees levied by the city with the permission of the state. The most important of these were incomes from the sale of spirits, wine, beer, the holding of markets and fairs, and the use of roads and railways. The fifth group included the income that arose after the pub law was acquired by the state in 1890: state compensation and various city tax supplements. Overall, revenues from urban property in the years 1870-1880 approached, and sometimes even exceeded, $60 \%$ of budget revenues. In the 1890 s, their proportion fell below $40 \%$, increased to nearly $50 \%$ by the turn of the century, and then gradually decreased to about $30 \%$ by 1914 . The result of urban wealth management has been future urbanization and infrastructure investiments, with the inevitable indebtedness at a disadvantage.
\end{abstract}

Keywords: city of Pécs, wealth management, budget, dualism, indebtedness

Die Städte mit Munizipialrecht funktionierten im Dualismus auf anderen Grundlagen, als die heutigen Städte, da sie autark waren. Die Städte verfügten über beachtliches Vermögen, womit sie selbst wirtschafteten, und auch der Staat überließ ihnen gewisse Einkünfte. Die besten Quellen für die Untersuchung der Vermögenswirtschaft sind die jährlichen Rechenschaften. Leider wurden die Rechnungshofsbücher der Stadt 1946 für eine Papiersammlung ausrangiert, so stehen nur die jährlichen Budgets zur Verfügung. Die Haushaltsbudgets erhielten nicht den Etat der verschiedenen Fonds und Stiftungen (Krankenhausfond, Waisenkassefond, Armenhausfond, Stiftung für die Armenanstalt, Stiftung Rudolfinum, usw.). Sinngemäß enthielten sie die Modifizierungen, welche im Laufe des Jahres angenommen wurden, die Zusatzbudgets und die Ausgaben außerhalb des Budgets, wie zum Beispiel die während des Jahres aufgenommen Anleihen, den Ver- und Ankauf von Immobilien. ${ }^{1}$

Ab 1873 wurde die Doppik auf Vorschlag des Hauptkassierers Iván Dévényi aufgehoben und die sogenannte kameralistische Kassenführung eingeführt. ${ }^{2} \mathrm{Ab}$ diesem Jahr bis 1898 geschah die Kassenverwaltung betreffende Rechnungsführung und die Führung der Tagebücher (Journale), die des Hauptbuches und die der anderen Bücher nach der sogenannten Kameralistik. Das heißt, dass die einzelnen Einnahmen und Ausgaben im Budget extra nacheinander aufgelistet und diese Rubriken nicht zusammengepaart wurden. Ab 1898 wurde die Doppik wieder eingeführt.

Zwischen 1872 und 1898 wurden die Einnahmen und die Ausgaben in fünf, ab 1898 in 10 Gruppen (Abteile) eingeteilt. Bis 1899 wurde in Gulden gerechnet, ab 1900 in Krone. Im Interesse der Vergleichbarkeit rechneten wir den Gulden in Krone um, 1 Gulden entsprach 2 Kronen. Da sich die Gruppen und Items des Budgets mehrmals änderten, folgten wir im Laufe

\footnotetext{
1 Archiv des Komitats Baranya des Ungarischen Nationalarchivs (im Weiteren: MNL BaML) IV. 1412. Pécs Schriften des Städtischen Rechnungsamtes (im Weiteren: IV. 1412.) Budget der Stadt Pécs 1872-1918.; MNL BaML IV. 1402. a. Versammlungsprotokolle des Munizipialausschusses der Stadt Pécs (im Weiteren: IV. 1402. a.) 1872-1918.

2 MNL BaML IV. 1402. a. 323/1872. (20. Dezember)
} 
unserer Untersuchung den sich jährlich ändernden Rubriken nicht, sondern summierten wir die Items nach der Gruppierung des Budgets von 1914. ${ }^{3}$

Die aus Budgeteinnahmen der Stadt resultierenden Summen wuchsen zwischen 1872 und 1914 auf das Achtfache, von 474.970,88 Kronen auf 3.813.189 Kronen. ${ }^{4}$

Die größte Gruppe der städtischen Einnahmen stammte aus Revenuen des Gemeindevermögens. Der wichtigste Bestandteil des städtischen Vermögens bedeuteten das Grund- und Gebäudevermögen. Die Stadt Pécs besaß am Anfang des Dualismus einen bedeutenden Grundbesitz, aber verhältnismäßig nur wenige und bescheidenen Wert verkörpernde Gebäude. Die wichtigste Komponente des Grundvermögens bildete der städtische Wald. Der andere beachtliche Grundbesitz war das Landgut Megyer (Acker, Weide, wirtschaftliche Gebäude), dessen Ausdehnung sich auf mehrere Hundert Joch belief.

Im Adressenverzeichnis für die Wirtschaft von 1895 steht, dass die Immobilien der Stadt Pécs an Grund 5.167 Katastraljoch ausmachten. Davon waren 149 Katastraljoch Acker, 13 Katastraljoch Garten, 3 Katastraljoch Weingarten, 368 Katastraljoch Weide, 4.262 Katastraljoch Wald und 342 Katastraljoch Gebiet, das nicht unter die Grundsteuer fiel. ${ }^{5}$

$\mathrm{Ab} 1898$ wurden die Revenuen aus dem städtischen Vermögen in folgende fünf Gruppen gegliedert: Einnahmen aus der Wirtschaft und Gebäuden der Stadt, der Waldwirtschaft, den Zinsen des Geldkapitals, den Rechten und Abgaben, bzw. aus Konsumzusatzsteuern und staatlichen Substituten. Die letzten zwei Gruppen zogen wir in unserer Untersuchung zusammen. (Tabelle 1.)

Tabelle 1a: Revenuen aus dem städtischen Vermögen zwischen 1872 und 1914 in Krone

\begin{tabular}{|c|c|c|c|c|c|c|}
\hline Jahr & II/A & II/B & II/C & II/D-E & II & I-X \\
\hline & $\begin{array}{c}\text { Wirtschaft } \\
\text { und } \\
\text { Gebäude }\end{array}$ & Waldwirtschaft & Zinsen & $\begin{array}{c}\text { Rechten, Abgaben, } \\
\text { Substitute }\end{array}$ & $\begin{array}{c}\text { städtiches } \\
\text { Vermögen } \\
\text { Teilsumma }\end{array}$ & $\begin{array}{c}\text { Alle } \\
\text { Revenuen }\end{array}$ \\
\hline 1872 & 31285,26 & 42161,40 & 68814,34 & 128563,12 & 270824,12 & 474970,88 \\
\hline 1878 & 35987,90 & 45475,52 & 77820,70 & 182910,16 & 342194,28 & 585624,10 \\
\hline 1882 & 32408,60 & 38321,10 & 87607,40 & 201918,32 & 360255,42 & 580767,78 \\
\hline 1887 & 39856,74 & 53714,34 & 60765,54 & 226590,30 & 380926,92 & 599750,22 \\
\hline 1892 & 50443,32 & 55259,78 & 96562,52 & 303404,06 & 505669,68 & 878447,42 \\
\hline 1898 & 83328,00 & 71432,00 & 77790,00 & 312000,00 & 544550,00 & 1549270,00 \\
\hline 1899 & 84230,00 & 80756,00 & 69600,00 & 320780,00 & 555366,00 & 1399174,00 \\
\hline 1900 & 83082,00 & 74086,00 & 69410,00 & 534972,00 & 761550,00 & 1659502,00 \\
\hline 1901 & 128402,00 & 75386,00 & 69000,00 & 529365,00 & 801153,00 & 1670410,00 \\
\hline 1902 & 132642,00 & 81948,00 & 68300,00 & 522565,00 & 805455,00 & 1696390,00 \\
\hline 1903 & 144906,00 & 81051,00 & 99000,00 & 524665,00 & 849622,00 & 1820557,00 \\
\hline 1904 & 129880,00 & 74721,00 & 94800,00 & 518115,00 & 817516,00 & 1810388,00 \\
\hline 1905 & 145390,00 & 69947,00 & 92800,00 & 513025,00 & 821162,00 & 1856674,00 \\
\hline 1906 & 142490,00 & 76862,00 & 85000,00 & 523825,00 & 828177,00 & 1873414,00 \\
\hline
\end{tabular}

3 Es sind verschiedene, von einander leicht abweichende Versionen (Rechnungsamt, Stadtrat, Vollversammlung) der jährlichen städtischen Budgets erhalten. Mit Ausnahme des Ratsbudget von 1898 verwendeten wir immer die Budgets der Vollversammlung.

4 Ab 1908 wurden die nach den Darlehen zu zahlenden Zinsen und die Raten bei der Ausgabe auch zweimal aufgenommen: zusammengezogen und auch bei den einzelnen Items. Diese doppelt registrierten Items wurden als Rückerstattung auch im Abschnitt „Schulden” der Einnahmen angegeben.

5 A Magyar Korona országainak mezőgazdasági statisztikája. II. Gazdacímtár. Magyar Kir. Központi Statisztikai Hivatal, Bp. 1897. II. S. 74-75. 


\begin{tabular}{|c|c|c|c|c|c|c|}
\hline 1907 & 160020,00 & 91756,00 & 71800,00 & 522085,00 & 845661,00 & 1899561,00 \\
\hline 1908 & 160830,00 & 70311,00 & 71200,00 & 566647,00 & 868988,00 & 2155511,00 \\
\hline 1909 & 163186,00 & 79668,00 & 71000,00 & 581229,00 & 895083,00 & 2261115,00 \\
\hline 1910 & 169498,00 & 99313,00 & 71500,00 & 558875,00 & 899186,00 & 2348469,00 \\
\hline 1911 & 177714,00 & 109323,00 & 71600,00 & 584202,00 & 942839,00 & 2583914,00 \\
\hline 1912 & 179332,00 & 99454,00 & 72000,00 & 631331,00 & 982117,00 & 2658467,00 \\
\hline 1913 & 197507,00 & 114228,00 & 72000,00 & 663089,00 & 1046824,00 & 3190955,00 \\
\hline 1914 & 204350,00 & 146769,00 & 73500,00 & 708089,00 & 1132708,00 & 3813189,00 \\
\hline
\end{tabular}

Tabelle 1b: Revenuen aus dem städtischen Vermögen zwischen 1872 und 1914 in Prozent

\begin{tabular}{|c|c|c|c|c|c|c|}
\hline Jahr & II/A & II/B & II/C & II/D-E & II & I-X \\
\hline & $\begin{array}{c}\text { Wirtschaft } \\
\text { und } \\
\text { Gebäude }\end{array}$ & Waldwirtschaft & Zinsen & $\begin{array}{c}\text { Rechten, } \\
\text { Abgaben, } \\
\text { Substitute }\end{array}$ & $\begin{array}{c}\text { städtiches } \\
\text { Vermögen } \\
\text { Teilsumma }\end{array}$ & $\begin{array}{c}\text { Alle } \\
\text { Revenuen }\end{array}$ \\
\hline 1872 & 6,6 & 8,9 & 14,5 & 27,1 & 57,0 & 100,0 \\
\hline 1878 & 6,1 & 7,8 & 13,3 & 31,2 & 58,4 & 100,0 \\
\hline 1882 & 5,6 & 6,6 & 15,1 & 34,8 & 62,0 & 100,0 \\
\hline 1887 & 6,6 & 9,0 & 10,1 & 37,8 & 63,5 & 100,0 \\
\hline 1892 & 5,7 & 6,3 & 11,0 & 34,5 & 57,6 & 100,0 \\
\hline 1898 & 5,4 & 4,6 & 5,0 & 20,1 & 35,1 & 100,0 \\
\hline 1899 & 6,0 & 5,8 & 5,0 & 22,9 & 39,7 & 100,0 \\
\hline 1900 & 5,0 & 4,5 & 4,2 & 32,2 & 45,9 & 100,0 \\
\hline 1901 & 7,7 & 4,5 & 4,1 & 31,7 & 48,0 & 100,0 \\
\hline 1902 & 7,8 & 4,8 & 4,0 & 30,8 & 47,5 & 100,0 \\
\hline 1903 & 8,0 & 4,5 & 5,4 & 28,8 & 46,7 & 100,0 \\
\hline 1904 & 7,2 & 4,1 & 5,2 & 28,6 & 45,2 & 100,0 \\
\hline 1905 & 7,8 & 3,8 & 5,0 & 27,6 & 44,2 & 100,0 \\
\hline 1906 & 7,6 & 4,1 & 4,5 & 28,0 & 44,2 & 100,0 \\
\hline 1907 & 8,4 & 4,8 & 3,8 & 27,5 & 44,5 & 100,0 \\
\hline 1908 & 7,5 & 3,3 & 3,3 & 26,3 & 40,3 & 100,0 \\
\hline 1909 & 7,2 & 3,5 & 3,1 & 25,7 & 39,6 & 100,0 \\
\hline 1910 & 7,2 & 4,2 & 3,0 & 23,8 & 38,3 & 100,0 \\
\hline 1911 & 6,9 & 4,2 & 2,8 & 22,6 & 36,5 & 100,0 \\
\hline 1912 & 6,7 & 3,7 & 2,7 & 23,7 & 36,9 & 100,0 \\
\hline 1913 & 6,2 & 3,6 & 2,3 & 20,8 & 32,8 & 100,0 \\
\hline 1914 & 5,4 & 3,8 & 1,9 & 18,6 & 29,7 & 100,0 \\
\hline
\end{tabular}

Quelle: MNL BaML IV. 1412. 1872-1914.; IV. 1402. a. 1872-1913.

Das Wachstum der Revenuen der städtischen Wirtschaft, Gründe und Gebäude zwischen 1872 und 1914 blieb hinter dem Wachstum des Budgets. Es wuchs auf das Sechseinhalbfache, von 31.285,26 Kronen auf 204.350 Kronen. Die Einnahmen aus der Hauswirtschaft und den Immobilien bewegten sich um 6-7 \% des Budgets.

Die Stadt verwaltete ihre Gründe und Immobilien teilweise selbst, teilweise wurden sie verpachtet. Der größte Teil des Landgutes Megyer war in städtischer Verwaltung. Das Zentrum der städtischen Wirtschaft war die städtische Meierei, hier waren die Pferdegespanne. Laut 
des Vermögensinventars vom 1872 werde die 22 5/8 Joch große städtische Weide „selbst berwirtschaftet, es wächst darauf 500-700 Zentner Heu, 50-70 Zentner Grummet. Dieses Heu und Grummet werden von den Pferden und Stieren gefressen." Im Viehstall waren 10 Pferde und 7 Stiere. Laut des Adressenverzeichnisses für die Wirtschaft von 1895 dienten in der Meierei 15 Knechten, es wurde 9 Hornvieh und 29 Pferde gehalten, unter den wichtigeren Maschinen und Geräten war eine Sichtmaschine, 3 Pflüge, 2 Eggen, 1 Rolle, 16 Fuhrwerke.

Die Revenuen der Hauswirtschaft kamen in erster Linie aus dem Verkauf von Schafen, Pferden und Mist. Die Fuhrgebühr der hauptsächlich zur Bewässerung, Mülltransport, Beförderungen gebrauchten Gespanne erschien auf der anderen Seite als Ausgabe. Die Stadt konnte noch aus dem kommunalen Schlachthof, aus dem 1896 errichteten amerikanischen Rebschule und bis 1891 aus der städtischen Ziegelei mit Einnahmen rechnen.

Der größte Teil der Bodengebühren ergab sich durch die Verpachtung der durch die Stadt nicht bewirtschafteten Flächen des Landgutes Megyer. Die Stadt erhielt zum Beispiel 1907 nach 78,5 Joch Acker in Megyer 2.474,80 Kronen. In diesem Jahr gebührten der Stadt für weitere 66 Gründe, Territorien kleinere-größere Entgelte und Nutzungsgebühren. Für die Benutzung der Weiden der Budaer und Szigeter Vorstadt zahlten die interessierten Besitzer nach Zahl des ausgetriebenen Viehs und Tierart Weidepachtgebühren.

Von den Haus- und Geschäftsmieten waren die der auf dem Széchenyi-Platz liegenden Anwesen bedeutend, da diese große Anziehungskraft für das städtische Publikum hatten. Die Wochenmärkte, die großen Märkte wurden nämlich bis Anfang der 1920er Jahre hier, auf dem Hauptplatz abgehalten. ${ }^{6}$ Durch die Verpachtung des Wirtshauses am Balokány kam dagegen wenig Einnahme.

Die Stadt war gestrebt, nach Möglichkeiten ihre Immobilien zu vermehren, es wurden nur jene Immobilien verkauft, welche nicht nutzbar gemacht werden können. Unter anderen wurden 1891 die städtische Ziegelei für 5.005 Fl. ${ }^{7}$ verkauft, bzw. jener Teil der Immobilien, welche zur Erwerbung des Wasserrechts an der Tettye gekauft wurden, aber unbrauchbar waren.

Auch der Betriebszweig Waldwirtschaft wurde von der Stadt selbst betrieben. Der städtische Wald sicherte in erster Linie durch Verkauf von Bauholz, Langholz und Brennholz Einnahmen der Stadt. Seine Bedeutung war aber viel größer, da er das Naherholungsgebiet und touristisches Ziel für die Bürgerinnen und Bürger bedeutete, besonders ab 1892, als der Mecsek Verein e.V. gegründet wurde. Der Wald spielte auch in den Einnahmen der städtischen Einrichtungen und Angestellten eine Rolle, da ihnen für ihre Dienste auch Brennholz gebührte.

Der städtische Wald hatte auch aus sozialem Aspekt Bedeutung, da die ärmsten Schichten kostenloses Brennholz (Abfallholz) erhielten.

Die sich im Wald befindlichen verpachteten Steinbrüche und Sandgruben brachten jährlich 2.000-3.000 Kronen ein, die Waldweiden und das Kastaniensammeln sicherten dagegen nur wenig Einnahmen. Die städtischen Kohlengruben „István” und „Mátyás” im Lámpásvölgy brachten ab den 1910er Jahren größere Einkünfte. Sie wurden ab dem 1. September 1909 dem Grafen Károly Kornis verpachtet, der sie nächstes Jahr in Betrieb setzte. ${ }^{8}$ Es wurde 1910 mit 10.000, 1911 mit 16.212 und 1914 mit 26.832 Kronen Einnahme gerechnet.

Die Einkünfte der Waldwirtschaft wuchsen zwischen 1872 und 1914 etwa um das Dreifache, von 42.161,4 Kronen auf 146.769 Kronen. Ab den 1890er Jahren sank seine Rolle unter den Einnahmen des Budgets, in den 1870er und 1880er war diese Einnahme um $9 \%$, in den 1900er und 1910er Jahren um 4-5\%.

\footnotetext{
6 Dankó Imre: Adalékok a pécsi piacok és vásárok néprajzához. In: A Janus Pannonius Múzeum Évkönyve 9 (1964). Janus Pannonius Múzeum, Pécs, 1965. S. 169-186.

7 MNL BaML IV. 1402. a. 162/1890. (27. Oktober), 56/1891. (27. Mai), 136/1891. (29. Oktober)

8 Pécs város szénbányászata. A Bánya, 5. Dezember 1909, S. 5.
} 
Die Einnahmen des städtischen Geldkapitals, die Zinsen der Wertpapiere und Einlagen veränderten sich zwischen 1872 und 1914 nicht maßgebend.1872 beliefen sie sich auf 68.814,28 Kronen, 1914 auf 73.500 Kronen.

Das Rückgrat des städtischen Geldkapitals bildete der Kaufpreis der 500 ungarisches Joch (mit 1200 Quadratklaftern gerechnet) großen Steinkohlengrube, welches 1857 von der Donaudampfschifffahrtsgesellschaft gekauft wurde. Die DDSG war verpflichtet, in Raten 1 Million Fl. für das wertvolle Gebiet zu zahlen. ${ }^{9}$

Nach dem Vermögensinventar 1872 verfügte die Stadt Pécs insgesamt über 979.827 F1. 77 Kr. Kapital, dessen jährliche Zinsen 34.407 Fl.17 Kr. waren:

a). Staatsobligation $10.000 \mathrm{Fl}$., mit 5 \% Jahreszins: $420 \mathrm{Fl}$;

b). Obligation bei der DDSG im Wert von 968.100 Fl. mit 3 1/2 \% Jahreszins: $33.883 \mathrm{Fl} .50 \mathrm{Kr}$;

c). Anleihe bei 25 Pécser Bewohner im Wert von 1.727 Fl. 77 Kr. mit 6\% Jahreszins: $103 \mathrm{~F} 167 \mathrm{Kr}$.

Der Zins der städtischen Kapitalgelder wuchs in den 1870er Jahren und in der ersten Hälfte der 1880er Jahre auf 80-90 Tausend Kronen an. Danach sanken die Zinsen Jahr für Jahr, da die Stadt ihr Kapital auflebte, weil sie überwiegend mit Darlehen aus dem eigenen Stammkapital die Investitionen deckte. Die Stadt nahm bis 1898 insgesamt 1.286.512 Fl. 83 Kr. Darlehen aus dem Stammkapital auf, wovon sie im Laufe des Wirtschaftsprogramms 1898315.932 Fl. zurückzahlte. Auf Anordnung des Innenministers musste sie die 854.185 Fl. 39 Kr. Schulden während 63 Jahre zurückzahlen.

Mit der Erlösung des Ausschankrechtes erhielt die Stadt eine sog. Regaleentschädigungsobligation im Wert von 703.150 Fl. derer $4 \frac{1}{2} \%$ Jahreszins 31641 Fl. 75 Kr. Einnahme brachte, ${ }^{10}$ die etliche Jahre hindurch in den 1890er 100.000 Kronen Zins bedeutete. Bis 1898 sank der Zins des Geldkapitals auf eine Summe um die 70.000. Er wuchs in diesem Zeitalter nicht mehr bedeutend.

Wegen der Obigen spielte das Geldkapital eine abnehmende Rolle im städtischen Budget: in den 1870er und 1880er Jahren deckte es 12-15 \% der Einkünfte, in den 1890er 1900er Jahren bloß nur 4-5 \%, 1914 stürzte es auf $2 \%$.

$\mathrm{Zu}$ den größten Einnahmequellen der Stadt gehörten jene Rechte und Gefälle, welche der Staat den einzelnen Gemeinden überließ. Davon bedeuteten den größten Teil die Ausschankrechte: das Recht auf Ausschank von Schnaps und alkoholischen Getränken, der Bier- und das Weinzoll, und der Zoll auf die dörflichen Weine.

Das Recht auf Standgeld, Pflasterzoll, Pflasterzoll der Eisenbahn und die Grundübertragungsgebühren brachten größere Einkünfte. Eine kleinere Einnahme resultierte aus der Verpachtung des Jagdrechtes im Wald und des Jagdrechtes in der unteren Flur, Brückenwaage und Eichung (Markierung der Fässer).

Das Wirtshausrecht gehörte früher zu den den Grundherren zustehenden sogenannten kleineren königlichen wirtschaftlich nutzbaren Hoheitrechten (Regalen). Das sicherte der Stadt ausschließliches Recht auf Ausschank von allen alkoholischen Getränken und Eröffnung von Wirtshäusern und Gasthöfen. Die Stadt Pécs machte dieses Recht mit Verpachtung nutzbar. Die staatliche Erlösung des Ausschankrechtes 1890 betraf die Stadt sensibel, da sie ihr jährlich mehr als 6.500 Fl. Verlust verursachte. ${ }^{11}$ Auch das Zollrecht auf dörfliche Weine konnte die Stadt nur einstweilen, bis Ende der 1890er Jahre ausüben.

9 MNL BaML IV. 1420. Pécs város okmánytára Nr. 379.

10 MNL BaML IV. 1402. a. 3/1890. (jan. 22.); IV. 1412. Budget 1891.

11 MNL BaML IV.1406. o. Pécs Város Tanácsának különkezelt iratai 15. kcs. - Der Brief von János Aidinger an die Vollversammlung der Stadt Pécs. Um 1892. 
Zur Ersetzung der durch Entzug der Konsumsteuer ausfallenden Einnahmen sicherte der Staat eine einmalige Regaleentschädigungsobligation im Wert von 703.150 Fl. Außerdem zahlte er der Stadt jährlich Schadenersatz, in den Jahren 1891-1892 7.200 F1. (14.800 Kronen) die ab der Jahrhundertwende immer höher wurde. Überdies bestimmte eine sich zum Gesetz VI./1899 knüpfende Verordnung des Finanzministeriums, dass die Stadt aus der netto Einnahme des staatlichen Ausschankrechts jährlich 31.485,34 Kronen Beteiligung erhielt.

Darüber hinaus machte die Vollversammlung der Stadt Brauch aus ihrem Recht, als Gemeindeselbstverwaltung örtliche Staute zu verabschieden, und auferlegte ab 1891 der Bevölkerung auch Konsumzusatzsteuer. Die Vollversammlung verabschiedete den Widerstand der Bevölkerung besiegend in den 1890er Jahren die Staute über die Sodawasser- und Mineralensteuer, nach Wein, Fleisch und Branntwein zu zahlende städtische Konsumzusatzsteuer und Hauszinssteuer. Der Auferlegung von Konsumzusatzsteuern setzte die finanzielle Belastbarkeit der Bevölkerung Grenzen. Im Sinne des 1899 über den Hauszins verabschiedeten Statuts wurde nach jeder Lokalität (Wohnung, Geschäft) $5 \%$ des Mietwertes, d.h. pro Gulden 5 Kreuzer Steuer von den Bewohnern, bzw. Besitzern eingenommen. Diese Steuerart musste auch von den sonst steuerfreien Offizieren, Munizipialbeamten, Angestellten, Dienern, usw. bezahlt werden. Die Einführung des Statuts über die Hauszinssteuer löste einen derartig großen Protet aus, dass die Versammlung des Munizipialausschusses gemüßigt war, es zu verändern. Nach dem neuen Statut von 1901 wurde die Steuer nicht mehr den Bewohnern, sondern den Besitzern auferlegt und sie wurde auf $3 \%$ ermäßigt, also es wurden nach 1 Kronenwert 3 Heller eingenommen. ${ }^{12}$

Nach der staatlichen Erlösung des Ausschankrechtes nahm die Stadt die betrachtlichen Rechte und Gefälle in eigene Regie. Die Brückenwaage und die Eichung wurden von Anfang an von der Stadt verwaltet. Die Stadt nahm den Pflasterzoll und den Pflasterzoll der Eisenbahn ab dem 1. Juli 1898 in die eigenen Hände. ${ }^{13}$ Nach der Maßnahme erhob die Versammlung des Munizipialausschuss der Stadt die Gebühren. So wuchsen die Einnahmen aus den Zöllen und Standgeldern zwischen 1897 und 1900 um 76 \%, von 132.524 Kronen auf 233.328 Kronen.

Die Belastbarkeit der Bevölkerung setzte auch hier Schränke der weiteren Erhöhung, da sich diese Einnahmen in den 1900er kaum veränderten und sie bis 1914 nur auf 274.000 Kronen wuchsen.

Die Stadt verpachtete ausschließlich das Jagdrecht im Wald und das in der unteren Flur, welche in Eigenverwaltung nicht zu handhaben waren und ihre Rentabilität nicht zu erhöhen war. 1887 entstammten diesen Quellen 1.222 Kronen, 1914 1.504 Kronen Einnahmen.

Die aus den Rechten, Gefällen, dem staatlichen Schadenersatz und den städtischen Zusatzsteuern eingeflossene Einnahme wuchs zwischen 1872 und 1914 auf das Fünfeinhalbfache an, von 128.563,12 Kronen auf 708.089 Kronen. Das Wachstum wurde zwar von der Verstaatlichung des Ausschankrechtes gebrochen, aber es wurde ab der Jahrhundertwende durch die Auferlegung von städtischen Zusatzsteuern und Eigenverwaltung ausgeglichen. Ihre Proportion im Budget bewegte sich in den 1870er und 1880er Jahren um 30\%, in den 1890er sank sie auf Werte um $20 \%$ ab. Um die Jahrhundertwende erreichte sie zwar die 30 Prozent, aber bis 1914 fiel sie wieder unter $20 \%$.

Die Gesamteinnahmen aus dem städtischen Vermögen näherten sich in den 1870er und 1880er Jahren an die $60 \%$ des Budgets an, bzw. überschritten manchmal diese Proportion. In den 1890er Jahren sank ihre Proportion unter $40 \%$, um die Jahrhundertwende wuchs sie fast auf $50 \%$ an, danach sank sie bis 1914 stufenweise etwa unter $30 \%$.

12 MNL BaML IV. 1418. Pécs város szabályrendeleteinek levéltári gyüjteménye 55. sz. Pécs szab. kir. város szabályrendelete a házbérfillérek beszedése és kezelése tárgyában.; Pilkhoffer, Mónika: Pécs építészete a századfordulón (1888-1907). (Pannónia Könyvek.) Pro Pannonia Kiadói Alapítvány, Pécs, 2004. S. 119.

13 MNL BaML IV.1402. a. 105/1898. (13. Juni) 
Die Ausgaben wurden gleich den Einnahmen zwischen 1872 und 1898 in 5 Gruppen (Abteilungen) geteilt, ab 1898 in 10 Gruppen. (Tabelle 2.)

Tabelle 2: Die Ausgaben des Budgets in Krone und Prozent zwischen 1872 und 1914.

\begin{tabular}{|c|c|c|c|c|c|c|}
\hline Jahr & I & II & & I & II & \\
\hline & $\begin{array}{c}\text { Verwaltung } \\
\text { (in Krone) }\end{array}$ & $\begin{array}{c}\text { Vermögen } \\
\text { der Stadt } \\
\text { (in Krone) }\end{array}$ & $\begin{array}{c}\text { Gesamtausgaben } \\
\text { (in Krone) }\end{array}$ & $\begin{array}{c}\text { Verwaltung } \\
\text { (in \%) }\end{array}$ & $\begin{array}{c}\text { Vermögen } \\
\text { der Stadt } \\
\text { (in \%) }\end{array}$ & $\begin{array}{c}\text { Gesamtausgaben } \\
\text { (in \%) }\end{array}$ \\
\hline 1872 & 174134,72 & 123388,30 & 474970,88 & 36,7 & 26,0 & 100,0 \\
\hline 1878 & 214547,38 & 138468,44 & 585624,10 & 36,6 & 23,6 & 100,0 \\
\hline 1882 & 234546,64 & 142115,26 & 580767,78 & 40,4 & 24,5 & 100,0 \\
\hline 1887 & 252480,80 & 121498,70 & 599750,22 & 42,1 & 20,3 & 100,0 \\
\hline 1892 & 302264,52 & 114163,90 & 878447,42 & 34,4 & 13,0 & 100,0 \\
\hline 1898 & 269648,00 & 214300,00 & 1549270,00 & 17,4 & 13,8 & 100,0 \\
\hline 1899 & 295586,00 & 244626,00 & 1399124,00 & 21,1 & 17,5 & 100,0 \\
\hline 1900 & 393730,00 & 282226,00 & 1659502,00 & 23,7 & 17,0 & 100,0 \\
\hline 1901 & 379018,00 & 326998,00 & 1669435,00 & 22,7 & 19,6 & 100,0 \\
\hline 1902 & 379960,00 & 342949,00 & 1695968,00 & 22,4 & 20,2 & 100,0 \\
\hline 1903 & 388247,00 & 352753,00 & 1820542,00 & 21,3 & 19,4 & 100,0 \\
\hline 1904 & 389620,00 & 341151,00 & 1810388,00 & 21,5 & 18,8 & 100,0 \\
\hline 1905 & 396500,00 & 339248,00 & 1855474,00 & 21,4 & 18,3 & 100,0 \\
\hline 1906 & 411711,00 & 340210,00 & 1872042,00 & 22,0 & 18,2 & 100,0 \\
\hline 1907 & 418504,00 & 355483,00 & 1899055,00 & 22,0 & 18,7 & 100,0 \\
\hline 1908 & 435569,00 & 336114,00 & 2153006,00 & 20,2 & 15,6 & 100,0 \\
\hline 1909 & 459679,00 & 341462,00 & 2260663,00 & 20,3 & 15,1 & 100,0 \\
\hline 1910 & 474129,00 & 358145,00 & 2345376,00 & 20,2 & 15,3 & 100,0 \\
\hline 1911 & 573401,00 & 378625,00 & 2583009,00 & 22,2 & 14,7 & 100,0 \\
\hline 1912 & 586299,00 & 398057,00 & 2653911,00 & 22,1 & 15,0 & 100,0 \\
\hline 1913 & 649600,00 & 430140,00 & 3190885,00 & 20,4 & 13,5 & 100,0 \\
\hline 1914 & 713861,00 & 434282,00 & 3810552,00 & 18,7 & 11,4 & 100,0 \\
\hline
\end{tabular}

Quelle: MNL BaML IV. 1412. 1872-1914.; IV. 1402. a. 1872-1913.

Der Zuwachs der Verwaltungsausgaben zwischen 1872 und 1914 blieb weit unter dem Zuwachs des Budgets und auch ihre Proportion innerhalb des Budgets verbesserte sich. Die Verwaltungsgebühren wuchsen nur das Vierfache, von 174.134,72 Kronen auf 713.861 Kronen. Zwischen 1872 und 1892 wurde in Allgemeinen ein Drittel des Budgets für die Finanzierung der Stadtverwaltung, diese Proportion lag später um $20 \%$.

$\mathrm{Zu}$ den Betriebsausgaben kann man den mit den kommunalen Ausgaben (Beleuchtung, Müll) reduzierten Teil der Verwaltungsausgaben und die Ausgaben der Polizei rechnen. Die Besoldung der Angestellten der Stadtverwaltung und Vermögensbewirtschaftung, die Bekleidung des Dienstpersonals, die Renten, die Gnadengehälter, die Unterkunftmieten, der Bürobedarf, die Reisekosten, die Tagegelder, bzw. die Besoldung und Bekleidung der Polizei, die Wohnungskosten und andere Ausgaben der Polizei und die der Feuerwehr verzehrten riesige Summen. ${ }^{14}$

14 In die Ausgaben wurden bis 1898 auch die Ausgaben des Wirtschaftsbüros und des Personals des Gesundheitswesens aufgenommen. Ab 1898 wurden die Ersten unter der städtischen Vermögensbewirtschaftung 
Die Proportion der Ausgaben der städtischen Vermögensbewirtschaftung (Meierei und Gebäude, Waldwirtschaft, Rechte und Gefälle) gestaltete sich zwischen 1872 und 1914 im Budget günstiger. In den 1870er und1880er Jahren verwendete man noch ein Viertel des Budgets für die Vermögensbewirtschaftung. In den 1890er Jahren verminderte sich die Proportion auf 13-17 \%, im ersten Jahrzehnt der 1900er Jahre bewegte sie sich um 18-20 \%, ab 1908 verminderte sie sich allmählich auf $11 \%$.

Die Verschuldung der Stadt Pécs wurde wegen der Finanzierungsschwierigkeiten ähnlich anderen ungarischen Städten unvermeidlich. Die Verschuldung der Stadt stieg im Budget zwischen 1872 und 1914 spektakulär um das Siebzehnfache an, von 49.320,2 Kronen auf 838.100 Kronen.

Die Stadt Pécs verfügte auch schon in den ersten Jahren des Dualismus über hohe Schulden. Aufgrund des Vermögensinventars vom 1872 hatte die Stadt 281.308 F1. 92 Kr. Schulden. Sie überschritten die Ausgaben des Jahresbudgets (237.485 F1. 44 Kr.).

Von den Schulden waren 31.588 Fl. $10 \mathrm{Kr}$. Kassenrückstand, für den Bau des Krankenhauses wurde ein Darlehen von 34.300 Fl. aufgenommen, so musste es nicht die Stadtkasse tilgen, sondern die Krankenhauskasse. Nur etwa ein Fünftel, 19,02 \% (42.715 Fl. 5 Kr.) der Darlehenschuld (215.420 F1. 82 Kr.) war Bankschuld. Den größten Teil der Darlehen nahm die Stadt nicht von professionellen Geldinstituten auf, sondern von verschiedenen Privatpersonen. Die Kreditpolitik der Stadt folgte eher den Zuständen vor 1848.

Die Schulden der Stadt machten am 31. Dezember 1896 genau 2.619.407 F1. 30 Kr. aus:

1. Aus dem städtischen Stammkapital zwischen 1878 und 1894 aufgenommenen 1.271.512 F1. 83 Kr. Darlehen - 875.998 F1. 41 Kr.

2. Von kleineren Fonds und Stiftungen aufgenommenen Darlehen, welche keine Amortisationsdarlehen waren - 210.002 Fl. $83 \mathrm{Kr}$.

3. Die Gesamtkredit von der Pester Ungarischen Handelsbank, Stiftungskasse der PécserDiözese, Pécser Sparkasse, Pécs-Baranyaer Zentralsparkasse - 1533406 Fl. 6 Kr. ${ }^{15}$

Beinahe 60 \% der Kredite waren schon von Banken, aber man kann die Proportion der zinsfreien Darlehen, welche aus dem Stammkapital oder von Fonds und Stiftungen aufgenommen wurden, noch immer hoch bezeichnen. Aus den in den 1870er und 1890er Jahren aufgenommen Krediten wurden die Investitionen der Ära des Dualismus finanziert: der Aufbau der Schulen, Kindergärten, Kasernen, des Theaters, Krankenhauses, usw., die Gebäuderenovierungen, der Ausbau der Wasserleitung und der Ankauf der dazu nötigen Wasserrechte, der Kauf der Mühlen, die Eröffnung neuer Straßen, die Regulierung des Wasserlaufs „Pécsi víz", der Erwerb von Grundstücken und Häusern bzw. Dergleichen.

Bürgermeister Imre Majorossy arbeitete seines Wirtschaftsprogramms für das Jahr 1897 für die Konvertierung der Darlehen und Investitionen. Die Stadt konnte die sich auf 4,5 Millionen Kronen belaufende "großes Darlehen” nach mehrmaligen Versuchen endlich 1902 aufnehmen. Die Ungarische Hypothek Bank sicherte den Kredit mit einer Tilgungsdauer von 65 Jahren. ${ }^{16}$ Es war früher die allgemeine Auffassung, dass es ratsam ist, die Lasten der für mehrere Generationen geplanten Investitionen mit einem Darlehen mit langer Tilgungsdauer auf mehrere Jahrzehnte zu verteilen. ${ }^{17}$

Die Summe des „großen Darlehens” machte das Zweieinhalbfache (265 \%) des städtischen Budgets (1 697638 Kronen) für 1902 aus. Aus der Anleihe wurde die städtischen Schulden

\footnotetext{
abgerechnet, welche 1898 11.864 Kronen, 1914 20.231 Kronen ausmachten. Die Summe unter die Ausgaben des Gesundheitswesens gestellter Bezüge war 1898 6.208 Kronen, 1914 22.819 Kronen.

15 MNL BaML IV. 1406. b. Pécs Város Tanácsának közigazgatási iratai 10667/1897.

16 MNL BaML IV.1402. a. 49/1902. (márc. 20.)

17 Sipos, András: Várospolitika és városigazgatás Budapesten 1890-1914. (Várostörténeti tanulmányok.) Budapest Főváros Levéltára, Budapest, 1996. S. 131.
} 
mit ungünstigen Zinsen konvertiert, bzw. zurückgezahlt aus 2.150.000 Kronen wurden die geplanten Investitionen gedeckt. Am 31. Dezember 1901 beliefen sich die Schulden der Stadt auf 4.062.945 Kronen 22 Heller, wovon zwei große Kredite der Pester Ungarischen Handelsbank übrig blieben, welche zusammen 2.069.905 Kronen 66 Heller ausmachten. ${ }^{18}$ Nach dem Wirtschaftsprogramm von 1897 wurden die städtischen Investitionen aus Bankkrediten verwirklicht, was zu neuerer Verschuldung führte.

Der Innenminister verlangte 1916 Unterrichtung über die Darlehensschulden der Städte mit Munizipialrecht. In der Aufstellung I. mussten die Daten über Tilgungsdarlehen aufgenommen werden, in der Aufstellung II. die der Kontokorrentkredite. Die erste Aufstellung blieb leider nicht erhalten. Die Schuld der Stadt aus Kontokorrentkrediten machte am 21. Juni 1916 aufgrund der Aufstellung des Hauptrechnungsführers Kornél Deutsch genau 16.006.480 Kronen 56 Heller aus. In dieser Liste steht aber nicht jenes Girokontodarlehen mit 2.589.000 Kronen, welche die Stadt für den Kauf des Elektrizitätswerks aufnahm, weil die Raten nicht die Stadt, sondern die Pécser Elektrizitätswerk AG. zahlte. Auch die Darlehen für das Krankenhaus fehlen, weil die Tilgung Aufgabe der Krankenhauskasse war. Gleichfalls fehlt das für die Sicherung der Verpflegung aufgenommene Girokontodarlehen, da dessen Zinsen vom Verpflegungsüberfluß getragen wurden und das Kapital durch den Schweinebestand, dessen Wert über 2 Millionen Kronen lag, und die Vorräte gesichert war. ${ }^{19}$

Die Bilanz kann doch als positiv bezeichnet werden, da die infrastrukturellen Investitionen und die im Interesse der Urbanisierung doch der Zukunft dienten.

Nach dem Ersten Weltkrieg infolge der gewaltigen Inflation entwerteten sich mit dem Geld auch die Schulden. ${ }^{20}$

In den Jahren 1847 und 1848 standen 17 Gebäude im Besitz der Stadt. ${ }^{21}$ Bis zum Anfang des Dualismus stieg die Zahl der städtischen Gebäude auf 37 an: das Rathaus, das Krankenhaus, Kasernen, Schulen, Pfarrhäuser, Meiereien, Zollhäuser und andere Gebäude, derer Wert auf 586.678 Fl. 30 1/2 Kr. geschätzt wurde. ${ }^{22} 1900$ besaß die Stadt Pécs aus den 8.763 Gebäuden der Gemeinde schon 171. Zwischen 1879 und 1908 wurden Investitionen in einem Wert von 13.197.000 Kronen durchgeführt, 39 \% aus eigenen Quellen, das Übrige aus Darlehen. ${ }^{23}$

Alles in allem wuchs der Wert der städtischen Immobilien zwischen 1872 und 1914 auf mehr als um das Dreizehnfache, von 1,5 Millionen Kronen auf 15,69 Millionen Kronen, und mehr als die Hälfte des städtischen Vermögens machten Immobilien aus. (Tabelle 3.)

Tabelle 3: Vermögensinventar der Stadt Pécs 1872 und 1912 in Krone und Prozent

\begin{tabular}{|l|c|c|c|c|}
\hline & 1872 (in Krone) & 1912 (in Krone) & 1872 (in \%) & 1912 (in \%) \\
\hline Immobilien & $1.174 .156,60$ & $15.693 .695,87$ & 23,2 & 50,5 \\
\hline Rechte und Gefälle & $1.811 .096,20$ & $10.596 .264,00$ & 35,7 & 34,1 \\
\hline Naturprodukte, Materialien & $5.737,36$ & $62.011,80$ & 0,0 & 0,2 \\
\hline Nutzviehe & $2.320,00$ & $22.040,00$ & 0,0 & 0,0 \\
\hline Gerätschaft, Möbel & $10.611,00$ & $250.905,24$ & 0,2 & 0,8 \\
\hline $\begin{array}{l}\text { Stammvermögen, } \\
\text { Kapital, Darlehen }\end{array}$ & $1.959 .655,54$ & $2.918 .292,34$ & 38,6 & 9,4 \\
\hline
\end{tabular}

18 MNL BaML IV.1406. e. Pécs Város Tanácsának „C”, Gazdasági és Építésügyi ügyosztályának iratai C-84/1902. MNL BaML IV.1406. g. Pécs Város Tanácsa Főjegyzői ügyosztályának iratai E-16641-1916.

20 Melega, Miklós: A modern város születése. Szombathely infrastrukturális fejlődése a dualizmus korában. (Archívum Comitatus Castriferrei 5.) Vas Megyei Levéltár, Szombathely, 2012. S. 391-392.

21 Kajtár, Istuán: Önkormányzati élet Pécsett (1848-1918). Baranya 3. (1990) Nr. 2., S. 72.

22 MNL BaML IV. 1412. Das Budget vom Jahr 1872.

23 Kajtár, István: Önkormányzati élet, zitiertes Werk, S. 72-73. 


\begin{tabular}{|l|c|c|c|c|}
\hline Forderung (aktiver Rückstand) & $103.113,56$ & $544.785,70$ & 2,1 & 1,7 \\
\hline Kassenüberrest & $8.349,54$ & $53.707,49$ & 0,2 & 0,2 \\
\hline Fonds & $\ldots$ & $948.713,51$ & $\ldots$ & 3,1 \\
\hline Städtisches Vermögen insgesamt & $5.075 .039,80$ & $31.090 .415,95$ & 100,0 & 100,0 \\
\hline Darlehen & 430841,64 & $12.990 .290,49$ & & \\
\hline Schulden, Debet & $131.776,20$ & $379.455,11$ & & \\
\hline $\begin{array}{l}\text { Lasten (passives } \\
\text { Vermögen) insgesamt }\end{array}$ & $562.617,84$ & $13.369 .745,60$ & & \\
\hline Reinvermögen & $4.512 .421,96$ & $17.720 .670,35$ & & \\
\hline
\end{tabular}

Quelle: MNL BaML IV. 1412. 1872, 1912. 